\title{
Piecewise Legendre spectral-collocation method for Volterra integro-differential equations
}

\author{
Zhendong $\mathrm{Gu}$ and Yanping Chen
}

\begin{abstract}
Our main purpose in this paper is to propose the piecewise Legendre spectral-collocation method to solve Volterra integro-differential equations. We provide convergence analysis to show that the numerical errors in our method decay in $h^{m} N^{-m}$-version rate. These results are better than the piecewise polynomial collocation method and the global Legendre spectral-collocation method. The provided numerical examples confirm these theoretical results.
\end{abstract}

\section{Introduction}

The VIDEs (Volterra integro-differential equations) have many applications, such as the modeling of heredity effects [26], modern theory of hysteresis [24], population dynamics [25], financial mathematics [17], rheology and viscoelasticity [20], turbulent diffusion [32], wavepower hydraulics [11], capillary theory [10] and medicine [9]. There are many existing numerical methods for VIDEs, such as the finite element methods [20], Runge-Kutta methods [32], finite difference methods [33] and Taylor series methods [12]. The monograph by Brunner [4] contains a wealth of material on the theory and numerical methods for VIDEs, with the focus being on the basic theory of Volterra equations and the piecewise polynomial collocation methods and their convergence analysis.

Spectral methods receive considerable attention mainly due to their high accuracy. Tang et al. [23] proposed a Legendre spectral-collocation method to solve VIEs (Volterra integral equations) of the second kind whose kernels and solutions are sufficiently smooth. Chen and Tang [6-8] proposed and analyzed a Jacobi spectral-collocation approximation for the linear VIEs of the second kind with weakly singular kernels provided that the underlying solutions of the VIEs are sufficiently smooth. In [16], the Jacobi spectral-collocation method is extended to solve the VIEs with the Abel type kernels. The Legendre spectral Galerkin method is investigated in $[\mathbf{2 7}, \mathbf{3 1}]$ for VIEs. The spectral-collocation methods also attract the interest of those people who study the Volterra type integral and related functional differential equations

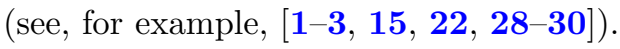

The VIDEs considered in this paper are as follows:

$$
\begin{gathered}
y^{\prime}(t)=a(t) y(t)+g(t)+\int_{0}^{t} K(t, s) y(s) d s, \quad t \in[0, T], \\
y(0)=y_{0} .
\end{gathered}
$$

We assume that the functions describing the above equations all possess continuous derivatives of at least order $m \geqslant 1$ on their respective domains, that is

Received 13 September 2013; revised 19 June 2014.

2010 Mathematics Subject Classification 65M70 (primary), 45D05, 45J05 (secondary).

This work is supported by National Science Foundation of China (91430104, 11271145), Specialized Research Fund for the Doctoral Program of Higher Education (20114407110009), and Foundation of Guangdong University of Finance (No. 14XJ03-08). The Corresponding Author of this paper is Yanping Chen. 


$$
a(t), g(t) \in C^{m}([0, T]), \quad K(t, s) \in C^{m}(\Omega), \quad \Omega:=\{(t, s): 0 \leqslant s \leqslant t \leqslant T\} .
$$

Theorem 3.1.4 in [4] shows that $y(t)$ lies in $C^{m+1}([0, T])$.

In this paper, we propose a piecewise Legendre spectral-collocation method to solve VIDEs (1.1). In our method, we change the definition domain $[0, T]$ to the standard interval $[-1,1]$, divide the interval $[-1,1]$ into $M+1$ subintervals $\left[\eta_{\mu}, \eta_{\mu+1}\right], \mu=0,1, \ldots, M$. In each subinterval $\left[\eta_{\mu}, \eta_{\mu+1}\right]$ we set the $(N+1)$-point Legendre Gauss-Lobatto points $x_{i}^{\mu}, i=0,1, \ldots, N$ as the collocation points, corresponding to the local Lagrange basis polynomial of degree $N$. Our method is to find the approximation of the exact solution at the collocation points. The convergence analysis we provide for our method shows that the numerical errors decay in the rate $h^{m-1 / 2} N^{1 / 2-m}$ and $h^{m} N^{-m}$ in $L^{\infty}$ and $L^{2}$ norms respectively, where $h=\max \left\{h_{\mu} / 2\right.$ : $\left.h_{\mu}=\eta_{\mu+1}-\eta_{\mu}, \mu=0,1, \ldots, M\right\}$. Numerical examples are presented to confirm these theoretical results. The convergence rate of the numerical errors in our method depends not only on $N$ but also on $h$ and $m$. We give numerical examples to underline their roles one by one.

To compare our method to the piecewise polynomial collocation methods in [4], we briefly introduce the later methods here.

In the piecewise polynomial collocation methods [4], the interval $[0, T]$ is divided into $M+$ 1 subintervals $\left[t_{n}, t_{n+1}\right], n=0,1, \ldots, M$. In each subinterval $\left[t_{n}, t_{n+1}\right]$, the number of the collocation points is $m$. Eventually the corresponding local Lagrange basis polynomial is of degree $m$. Theorem 3.2.3 in [4] shows that the numerical errors decay in the rate $h^{\prime m}, h^{\prime}=$ $\max \left\{t_{n+1}-t_{n}: n=0,1, \ldots, M\right\}$.

Now we can compare our method to the piecewise polynomial collocation method. First, in our method, the approximation solution in subinterval is polynomial of degree $N$, which can be chosen as any sufficiently large positive integer. This is different from the one in the piecewise polynomial collocation method where the approximate polynomial is of degree $m$. The higher degree approximation solution in our method may possess better regularity for exact solutions. Second, the errors in our method decay at the rate $h^{m-1 / 2} N^{1 / 2-m}$ or $h^{m} N^{-m}$ which is much sharper than $h^{\prime m}$ in the piecewise polynomial collocation method. Example 2 with $m=3$ in this paper confirms this theoretical result. For the piecewise polynomial collocation method, $m$ is chosen as the number of the collocation parameters, while in our method we do not need to consider what the exact value of $m$ is.

In the globe Legendre spectral-collocation method [15], the interval $[0, T]$ is changed to the standard interval $[-1,1]$. The $N+1$ collocation points (three types Legendre Gauss points) are set into $[-1,1]$. The globe basis functions are the polynomials of degree $N$ in the interval $[-1,1]$. The convergence speed of the errors is $N^{1 / 2-m}$ in the norm $L^{\infty}$.

Comparing our method with the globe Legendre spectral-collocation method, we can know that both methods have the same degree of the basis polynomial function. The highlight for our method is that the errors decay faster than in the globe Legendre spectral-collocation method. We provide examples to illustrate this theoretical result (see Example 2 with $m=3$ ).

This paper is organized as follows. In $\S 2$, we introduce the Legendre spectral-collocation method for VIDEs. The existence of a solution to the discrete system is discussed in $\S 3$. Some useful lemmas for the convergence analysis will be provided in $\S 4$, and the convergence analysis, in both $L^{\infty}$ and $L^{2}$, will be given in $\S 5$. Numerical experiments are carried out in $\S 6$. Finally, in $\S 7$, we end with the conclusion and future work.

\section{Piecewise Legendre spectral-collocation method}

For ease of analysis we change the interval $[0, T]$ to the standard interval $[-1,1]$. Precisely, we use the variable transformation

$$
t(x)=\frac{T}{2}(x+1), \quad s(z)=\frac{T}{2}(z+1) .
$$


Then (1.1) can be written as

$$
\begin{gathered}
u^{\prime}(x)=A(x) u(x)+f(x)+\int_{-1}^{x} R(x, z) u(z) d z, \quad x \in[-1,1], \\
u(-1)=y_{0},
\end{gathered}
$$

where

$$
\begin{gathered}
u(x):=y(t(x)), \quad A(x):=\frac{T}{2} a(t(x)) \\
f(x):=\frac{T}{2} g(t(x)), \quad R(x, z):=\left(\frac{T}{2}\right)^{2} K(t(x), s(z)) .
\end{gathered}
$$

Divide the definition domain $[-1,1]$ into $M+1$ subintervals $\delta_{\mu}:=\left[\eta_{\mu}, \eta_{\mu+1}\right] \subset[-1,1]$, where $\eta_{0}=-1, \eta_{M+1}=1, h_{\mu}:=\eta_{\mu+1}-\eta_{\mu}, \mu=0,1, \ldots, M$. Set the collocation points as

$$
X_{N}:=\bigcup_{\mu=0}^{M} X^{\mu}, \quad X^{\mu}:=\left\{x_{i}^{\mu}: \eta_{\mu}=x_{0}^{\mu}<x_{1}^{\mu}<\ldots<x_{N}^{\mu}=\eta_{\mu+1}\right\}
$$

where

$$
x_{i}^{\mu}:=\frac{h_{\mu}}{2} x_{i}+\frac{\eta_{\mu+1}+\eta_{\mu}}{2} ;
$$

here $x_{i}, i=0,1, \ldots, N$ are the $(N+1)$-point Legendre Gauss-Lobatto points in the standard interval $[-1,1]$. Then $(2.2)$ holds at $x_{i}^{\mu}, i=0,1, \ldots, N, \mu=0,1, \ldots, M$,

$$
u^{\prime}\left(x_{i}^{\mu}\right)=A\left(x_{i}^{\mu}\right) u\left(x_{i}^{\mu}\right)+f\left(x_{i}^{\mu}\right)+\int_{-1}^{x_{i}^{\mu}} R\left(x_{i}^{\mu}, z\right) u(z) d z .
$$

We use $u_{i}^{\mu}$ to approximate $u\left(x_{i}^{\mu}\right), \rho_{i}^{\mu}$ to approximate $u^{\prime}\left(x_{i}^{\mu}\right)$. Then we can use

$$
u_{\mu}(x):=\sum_{j=0}^{N} u_{j}^{\mu} F_{j}^{\mu}(x), \quad x \in\left[\eta_{\mu}, \eta_{\mu+1}\right]
$$

to approximate $\left.u\right|_{\delta_{\mu}}(x)$, that is the restriction of $u(x)$ to the interval $\left[\eta_{\mu}, \eta_{\mu+1}\right]$. We denote by $F_{j}^{\mu}(x), x \in\left[\eta_{\mu}, \eta_{\mu+1}\right]$, the $j$ th Lagrange interpolation basic function associated with the collocation points $x_{0}^{\mu}, x_{1}^{\mu}, \ldots, x_{N}^{\mu}$ in the interval $\left[\eta_{\mu}, \eta_{\mu+1}\right]$. Similarly, we use

$$
\rho_{\mu}(x):=\sum_{j=0}^{N} \rho_{j}^{\mu} F_{j}^{\mu}(x), \quad x \in\left[\eta_{\mu}, \eta_{\mu+1}\right]
$$

to approximate $\left.u^{\prime}\right|_{\delta_{\mu}}(x)$, that is the restriction of $u^{\prime}(x)$ to the subinterval $\left[\eta_{\mu}, \eta_{\mu+1}\right]$. Eventually $u(x)$ can be approximated by

$$
u^{N}(x):=u_{\mu}(x) \text { if } x \in\left[\eta_{\mu}, \eta_{\mu+1}\right], \mu=0,1, \ldots, M,
$$

and $u^{\prime}(x)$ can be approximated by

$$
\rho^{N}(x):=\rho_{\mu}(x) \quad \text { if } x \in\left[\eta_{\mu}, \eta_{\mu+1}\right], \mu=0,1, \ldots, M .
$$

Then (2.5) can be approximated by

$$
\rho_{i}^{\mu} \approx A\left(x_{i}^{\mu}\right) u_{i}^{\mu}+f\left(x_{i}^{\mu}\right)+\int_{-1}^{x_{i}^{\mu}} R\left(x_{i}^{\mu}, z\right) u^{N}(z) d z
$$


which can be written as

$$
\rho_{i}^{\mu} \approx A\left(x_{i}^{\mu}\right) u_{i}^{\mu}+f\left(x_{i}^{\mu}\right)+\sum_{r=0}^{\mu-1} \int_{\eta_{r}}^{\eta_{r+1}} R\left(x_{i}^{\mu}, z\right) u_{r}(z) d z+\int_{\eta_{\mu}}^{x_{i}^{\mu}} R\left(x_{i}^{\mu}, z\right) u_{\mu}(z) d z .
$$

In order to compute the integral term by the Gauss quadrature rule, we change the integration interval to the standard interval $[-1,1]$. Note that the variable transformation

$$
z(a, b, v):=\frac{b-a}{2} v+\frac{b+a}{2}, \quad v \in[-1,1]
$$

can change the interval $[a, b]$ to $[-1,1]$. For simplicity, we denote

$$
z_{r}(v):=z\left(\eta_{r}, \eta_{r+1}, v\right), \quad v \in[-1,1], r \geqslant 0 .
$$

Using the Gauss quadrature formula to approximate the integration term in (2.7) we obtain that

$$
\begin{aligned}
\rho_{i}^{\mu}= & A\left(x_{i}^{\mu}\right) u_{i}^{\mu}+\sum_{r=0}^{\mu-1} \frac{h_{r}}{2} \sum_{k=0}^{N} R\left(x_{i}^{\mu}, z_{r}\left(v_{k}\right)\right) u_{r}\left(z_{r}\left(v_{k}\right)\right) \omega_{k} \\
& +\frac{h_{\mu}}{2} \frac{x_{i}+1}{2} \sum_{k=0}^{N} R\left(x_{i}^{\mu}, z_{\mu}\left(z\left(-1, x_{i}, v_{k}\right)\right)\right) u_{\mu}\left(z_{\mu}\left(z\left(-1, x_{i}, v_{k}\right)\right)\right) \omega_{k},
\end{aligned}
$$

where $v_{k}, k=0,1, \ldots, N$ are the $(N+1)$-point Legendre Gauss-Lobatto points in the standard interval $[-1,1]$, corresponding to the weights $\omega_{k}, k=0,1, \ldots, N$. Note that for $j, k=0,1, \ldots, N, r=0,1, \ldots, M$

$$
\begin{gathered}
F_{j}^{r}\left(z_{r}\left(v_{k}\right)\right)=F_{j}\left(v_{k}\right)= \begin{cases}1, & k=j, \\
0, & k \neq j,\end{cases} \\
F_{j}^{r}\left(z_{r}(z(-1, x, v))\right)=F_{j}(z(-1, x, v)),
\end{gathered}
$$

where $F_{j}(v)$ is the $j$ th Lagrange interpolation basic function associated with the $(N+1)$-point Legendre Gauss-Lobatto points in the standard interval $[-1,1]$. Then $(2.10)$ can be simplified as

$$
\rho_{i}^{\mu}=A\left(x_{i}^{\mu}\right) u_{i}^{\mu}+f\left(x_{i}^{\mu}\right)+\beta\left(x_{i}^{\mu}\right), \quad \mu=0,1, \ldots, M ; i=0,1, \ldots, N,
$$

where

$$
\begin{gathered}
\beta\left(x_{i}^{\mu}\right):=\sum_{r=0}^{\mu-1} \frac{h_{r}}{2} \beta_{1}^{r}\left(x_{i}^{\mu}\right)+\frac{h_{\mu}}{2} \frac{x_{i}+1}{2} \beta_{2}\left(x_{i}^{\mu}\right), \\
\beta_{1}^{r}\left(x_{i}^{\mu}\right):=\sum_{k=0}^{N} R\left(x_{i}^{\mu}, z_{r}\left(v_{k}\right)\right) u_{k}^{r} \omega_{k}, \quad r=0,1, \ldots, \mu-1, \\
\beta_{2}\left(x_{i}^{\mu}\right):=\sum_{j=0}^{N} u_{j}^{\mu} \sum_{k=0}^{N} R\left(x_{i}^{\mu}, z_{\mu}\left(z\left(-1, x_{i}, v_{k}\right)\right)\right) F_{j}\left(z\left(-1, x_{i}, v_{k}\right)\right) \omega_{k} .
\end{gathered}
$$

However, the linear system (2.12) alone is not enough to find out the unknown elements. We need another one linear system associated with $u_{i}^{\mu}, \rho_{i}^{\mu}, i=0,1, \ldots, N, \mu=0,1, \ldots, M$. 
Note that

$$
\begin{aligned}
u\left(x_{i}^{\mu}\right) & =u(-1)+\int_{-1}^{x_{i}^{\mu}} u^{\prime}(z) d z \\
& =y_{0}+\sum_{r=0}^{\mu-1} \frac{h_{r}}{2} \int_{-1}^{1} u^{\prime}\left(z_{r}(v)\right) d v+\frac{h_{\mu}}{2} \frac{x_{i}+1}{2} \int_{-1}^{1} u^{\prime}\left(z_{\mu}\left(z\left(-1, x_{i}, v\right)\right)\right) d v .
\end{aligned}
$$

Then we can approximate the above equation by

$$
u_{i}^{\mu}=y_{0}+\alpha\left(x_{i}^{\mu}\right), \quad \mu=0,1, \ldots, M ; i=0,1, \ldots, N,
$$

where

$$
\alpha\left(x_{i}^{\mu}\right)=\sum_{r=0}^{\mu-1} \frac{h_{r}}{2} \sum_{k=0}^{N} \rho_{k}^{r} \omega_{k}+\frac{h_{\mu}}{2} \frac{x_{i}+1}{2} \sum_{j=0}^{N} \rho_{j}^{\mu} \sum_{k=0}^{N} F_{j}\left(z\left(-1, x_{i}, v_{k}\right)\right) \omega_{k} ;
$$

(2.14) is another linear system we want to find.

The piecewise Legendre spectral-collocation method is to find $\rho_{i}^{\mu}, u_{i}^{\mu}, i=0,1, \ldots, N$, $\mu=0,1,2, \ldots, M$ which satisfy (2.12) and (2.14). The approximation to $y(t)$ is $u^{N}((2 / T) t-1)$, the approximation to $y^{\prime}(t)$ is $(2 / T) \rho^{N}((2 / T) t-1)$. An efficient computation of $F_{j}(s)$ can be found in $[\mathbf{5}]$ or $[\mathbf{2 3}]$.

\section{The existence of the solution to the discrete system}

In this section, we will discuss the existence of the solution to the discrete system (2.12) and (2.14) which is written in matrix form as

$$
\left\{\begin{array}{l}
U^{\prime(\mu)}=\Phi_{1}^{(\mu)}+A^{(\mu)} U^{(\mu)}+R_{1}^{(\mu)} U^{(\mu)} \\
U^{(\mu)}=\Phi_{2}^{(\mu)}+\frac{h_{\mu}}{2} R_{2}^{(\mu)} U^{\prime \mu} \\
\mu=0,1, \ldots, M
\end{array}\right.
$$

where

$$
\begin{gathered}
U^{(\mu)}:=\left[\rho_{0}^{\mu}, \rho_{1}^{\mu}, \ldots, \rho_{N}^{\mu}\right]^{\prime}, \\
U^{(\mu)}:=\left[u_{0}^{\mu}, u_{1}^{\mu}, \ldots, u_{N}^{\mu}\right]^{\prime}, \\
\Phi_{1}^{(\mu)}:=F^{(\mu)}+\sum_{r=0}^{\mu-1} R_{1}^{(r)} U^{(r)}, \quad \mu \geqslant 1, \\
\Phi_{2}^{(\mu)}:=y_{0}[1,1, \ldots, 1]^{\prime}+\sum_{r=0}^{\mu-1} R_{2}^{(r)} U^{(r)}, \quad \mu \geqslant 1, \\
F^{(\mu)}:=\left[f\left(x_{0}^{\mu}\right), f\left(x_{1}^{\mu}\right), \ldots, f\left(x_{N}^{\mu}\right)\right]^{\prime}, \\
A^{(\mu)}:=\operatorname{diag}\left[A\left(x_{0}^{\mu}\right), A\left(x_{1}^{\mu}\right), \ldots, A\left(x_{N}^{\mu}\right)\right], \\
R_{1}^{(r)}(i, k):=\frac{h_{r}}{2} R\left(x_{i}^{\mu}, z_{r}\left(v_{k}\right)\right) \omega_{k}, \quad r=0,1, \ldots, \mu-1, \\
R_{1}^{(\mu)}(i, j):=\frac{h_{\mu}}{2} \frac{x_{i}+1}{2} \sum_{k=0}^{N} R\left(x_{i}^{\mu}, z_{\mu}\left(z\left(-1, x_{i}, v_{k}\right)\right)\right) F_{j}\left(z\left(-1, x_{i}, v_{k}\right)\right) \omega_{k},
\end{gathered}
$$




$$
\begin{gathered}
R_{2}^{(r)}(i, k):=\frac{h_{r}}{2} \omega_{k}, \quad r=0,1, \ldots, \mu-1, \\
R_{2}^{(\mu)}(i, j):=\frac{h_{\mu}}{2} \frac{x_{i}+1}{2} \sum_{k=0}^{N} F_{j}\left(z\left(-1, x_{i}, v_{k}\right)\right) \omega_{k} .
\end{gathered}
$$

Plugging the second equation in (3.1) into the first one we obtain

$$
\left\{\begin{array}{l}
U^{\prime(\mu)}=\Phi_{1}^{(\mu)}+\frac{h_{\mu}}{2}\left(A^{(\mu)}+R_{1}^{(\mu)}\right) R_{2}^{(\mu)} U^{\prime \mu}+\left(A^{(\mu)}+R_{1}^{(\mu)}\right) \Phi_{2}^{(\mu)} \\
U^{(\mu)}=\Phi_{2}^{(\mu)}+\frac{h_{\mu}}{2} R_{2}^{(\mu)} U^{\prime \mu} \\
\mu=0,1, \ldots, M
\end{array}\right.
$$

This discrete system is based on the interval $\left[\eta_{\mu}, \eta_{\mu+1}\right]$. The existence of the solution to (3.2) depends on the existence of a solution to the first matrix equation of (3.2). Since $A(t), R(x, z), F_{j}(z)$ are continuous on their definition domain, the elements of matrix $A^{(\mu)}$, $R_{1}^{(\mu)}$ and $R_{2}^{(\mu)}, \mu=0,1, \ldots, M$, are all bounded. The Neumann lemma (see [19, p. 26], or $[4$, p. 87]) then shows that the inverse of the matrix

$$
\mathcal{B}^{(\mu)}:=I-\frac{h_{\mu}}{2}\left(A^{(\mu)}+R_{1}^{(\mu)}\right) R_{2}^{(\mu)}
$$

exists whenever

$$
\frac{h_{\mu}}{2}\left\|\left(A^{(\mu)}+R_{1}^{(\mu)}\right) R_{2}^{(\mu)}\right\|<1
$$

for some matrix norm. This clearly holds whenever $h_{\mu}$ is sufficiently small.

\section{Some useful lemmas}

In this section, we will provide some elementary lemmas, which are important for the derivation of error estimate in $\S 5$. In order to give the subsequent lemmas conveniently, we first introduce some spaces. For simplicity, we denote by $\partial_{x}^{k} u(x)$ the $k$ th derivative of $u$, that is $\partial_{x}^{k} u(x):=$ $\left(d^{k} u / d x^{k}\right)(x)$.

Let $(a, b)$ be a bounded interval of the real line. We denote by $L^{2}(a, b)$ the space of the measurable functions $u:(a, b) \rightarrow \mathbb{R}$ such that $\int_{a}^{b}|u(x)|^{2} d x<+\infty$. It is a Hilbert space for the inner product

$$
(u, v):=\int_{a}^{b} u(x) v(x) d x
$$

which induces the norm

$$
\|v\|_{L^{2}(a, b)}:=\left(\int_{a}^{b}|v(x)|^{2} d x\right)^{1 / 2} .
$$

Let $m \geqslant 1$ be an integer. We define $H^{m}(a, b)$ to be the vector space of the functions $v \in$ $L^{2}(a, b)$ such that all the distributions of $v$ of order up to $m$ can be represented by functions in $L^{2}(a, b)$. In short,

$$
H^{m}(a, b):=\left\{v \in L^{2}(a, b): \text { for } 0 \leqslant k \leqslant m, \partial_{x}^{k} v(x) \in L^{2}(a, b)\right\} .
$$

The space $H^{m}(a, b)$ is endowed with the inner product

$$
(u, v)_{m}=\sum_{k=0}^{m} \int_{a}^{b} \partial_{x}^{k} u(x) \partial_{x}^{k} v(x) d x
$$


for which $H^{m}(a, b)$ is a Hilbert space. The associated norm is

$$
\|v\|_{H^{m}(a, b)}:=\left((v, v)_{m}\right)^{1 / 2} .
$$

In bounding from the above approximation error, only some of the $L^{2}$-norms appearing on the right-hand side of the above norm enter into play. Thus, for a nonnegative integer $N$, it is convenient to introduce the semi-norm

$$
|v|_{H^{m ; N}(a, b)}:=\left(\sum_{k=\min (m, N+1)}^{m}\left\|\partial_{x}^{k} v(x)\right\|_{L^{2}(a, b)}^{2}\right)^{1 / 2},
$$

which implies that if $N \geqslant m-1$ then $|v|_{H^{m ; N}(a, b)}=\left\|\partial_{x}^{m} v\right\|_{L^{2}(a, b)}$.

The space $L^{\infty}(a, b)$ is the Banach space of the measurable functions $u$ that are bounded outside a set of measure zero, equipped with the norm

$$
\|u\|_{L^{\infty}(a, b)}:=\operatorname{ess}_{x \in(a, b)}|u(x)| .
$$

We denote by $C([a, b])$ the space of continuous functions on the interval $[a, b]$.

We define an interpolation operator $I_{N}$ associated with the collocation points $X_{N}$ as follows: for any continuous functions $v \in C([-1,1])$,

$$
I_{N} v(x):=I_{N}^{\mu}\left(\left.v\right|_{\delta_{\mu}}\right)(x) \quad \text { if } x \in \delta_{\mu}:=\left(\eta_{\mu}, \eta_{\mu+1}\right], \quad 0 \leqslant \mu \leqslant M,
$$

where $\left.v\right|_{\delta_{\mu}}(x)$ is the restriction of $v(x)$ to the subinterval $\left[\eta_{\mu}, \eta_{\mu+1}\right]$, and $I_{N}^{\mu}$ is the interpolation operator associated with the collocation points $X^{\mu}$ in the subinterval $\left[\eta_{\mu}, \eta_{\mu+1}\right]$, that is

$$
I_{N}^{\mu}\left(\left.v\right|_{\delta_{\mu}}\right)(x):=\left.\sum_{j=0}^{N} v\right|_{\delta_{\mu}}\left(x_{j}^{\mu}\right) F_{j}^{\mu}(x), \quad x \in\left[\eta_{\mu}, \eta_{\mu+1}\right] .
$$

Hereafter, $C$ denotes a generic positive constant that is independent of $N$.

From $[\mathbf{5}, \mathbf{1 3}, \mathbf{1 4}, \mathbf{1 8}, \mathbf{2 3}]$ we have the following lemma.

Lemma 4.1. Assume that $u \in H^{m}(-1,1), m \geqslant 1, v(x)$ is a bounded function. Then there exists a constant $C$ independent of $u$ and $v$ such that for $N \geqslant m-1$,

$$
\begin{gathered}
\left\|u-J_{N} u\right\|_{L^{2}(-1,1)} \leqslant C N^{-m}\left\|\partial_{x}^{m} u\right\|_{L^{2}(-1,1)}, \\
\left\|u-J_{N} u\right\|_{L^{\infty}(-1,1)} \leqslant C N^{(1 / 2)-m}\left\|\partial_{x}^{m} u\right\|_{L^{2}(-1,1)}, \\
\sup _{N}\left\|J_{N} v\right\|_{L^{2}(-1,1)} \leqslant C\|v\|_{L^{\infty}(-1,1)}, \\
\left\|J_{N}\right\|_{L^{\infty}(-1,1)} \leqslant \frac{2}{\pi} \log (N+1)+0.685,
\end{gathered}
$$

where $J_{N}$ is the interpolation operator associated with the $(N+1)$-point Legendre GaussLobatto points in the interval $[-1,1]$.

The above lemma will help us to deduce the following lemma.

Lemma 4.2. Assume that $u \in H^{m}(-1,1)$. Let $I_{N} u$ be the interpolation function defined in (4.1) where $N+1$ means the number of collocation points in the intervals $\delta_{\mu}:=\left[\eta_{\mu}, \eta_{\mu+1}\right], \mu=$ $0,1, \ldots, M$. Denote $h:=\max \left\{\left(h_{\mu} / 2\right): \mu=0,1, \ldots, M\right\}$. Then the following estimates hold for $N \geqslant m-1$,

$$
\left\|u-I_{N} u\right\|_{L^{2}(-1,1)} \leqslant C h^{m} N^{-m}\left\|\partial_{z}^{m} u\right\|_{L^{2}(-1,1)},
$$




$$
\begin{gathered}
\left\|u-I_{N} u\right\|_{L^{\infty}(-1,1)} \leqslant C h^{m-1 / 2} N^{(1 / 2)-m}\left\|\partial_{z}^{m} u\right\|_{L^{2}(-1,1)} \\
\left\|I_{N}\right\|_{L^{\infty}(-1,1)} \leqslant C \log (N+1) \\
\sup _{N}\left\|I_{N} u\right\|_{L^{2}(-1,1)} \leqslant C\|u\|_{L^{\infty}(-1,1)}
\end{gathered}
$$

Proof. By the definition of $I_{N}^{\mu}$ we know that $\left(I_{N}^{\mu}\left(\left.u\right|_{\delta_{\mu}}\right)\right)(z)$ is a function defined on the subinterval $\left[\eta_{\mu}, \eta_{\mu+1}\right]$. The variable transformation $z=z_{\mu}(v)$ changes it to be a function valued on the standard interval $[-1,1]$, that is

$$
\left(I_{N}^{\mu}\left(\left.u\right|_{\delta_{\mu}}\right)\right)\left(z_{\mu}(v)\right)=\left.\sum_{j=0}^{N} u\right|_{\delta_{\mu}}\left(x_{j}^{\mu}\right) F_{j}^{\mu}\left(z_{\mu}(v)\right)=\left.\sum_{j=0}^{N} u\right|_{\delta_{\mu}}\left(x_{j}^{\mu}\right) F_{j}(v), \quad v \in[-1,1] .
$$

The result (2.11) is used in the derivation of the second equality above. Moreover, we note that $\left.u\right|_{\delta_{\mu}}\left(z_{\mu}(v)\right)$ is a function defined on the interval $[-1,1]$. Its interpolation polynomial associated with the Legendre Gauss-Lobatto points $v_{j}, j=0,1, \ldots, N$ in the interval $[-1,1]$ is

$$
J_{N}\left(\left.u\right|_{\delta_{\mu}}\left(z_{\mu}(v)\right)\right)=\left.\sum_{j=0}^{N} u\right|_{\delta_{\mu}}\left(z_{\mu}\left(v_{j}\right)\right) F_{j}(v), \quad v \in[-1,1] .
$$

Note that $v_{j}=x_{j}$, then

$$
z_{\mu}\left(v_{j}\right)=x_{j}^{\mu}, \quad j=0,1, \ldots, N .
$$

Plugging this into the right-hand side of (4.11) yields

$$
J_{N}\left(\left.u\right|_{\delta_{\mu}}\left(z_{\mu}(v)\right)\right)=\left.\sum_{j=0}^{N} u\right|_{\delta_{\mu}}\left(x_{j}^{\mu}\right) F_{j}(v), \quad v \in[-1,1] .
$$

Combining (4.10) with (4.12) yields

$$
\left(I_{N}^{\mu}\left(\left.u\right|_{\delta_{\mu}}\right)\right)\left(z_{\mu}(v)\right)=J_{N}\left(\left.u\right|_{\delta_{\mu}}\left(z_{\mu}(v)\right)\right), \quad v \in[-1,1] .
$$

By (4.2), we have

$$
\begin{aligned}
& \int_{\eta_{\mu}}^{\eta_{\mu+1}}\left(\left.u\right|_{\delta_{\mu}}(z)-I_{N}^{\mu}\left(\left.u\right|_{\delta_{\mu}}\right)(z)\right)^{2} d z \\
& \quad=\frac{h_{\mu}}{2} \int_{-1}^{1}\left(\left.u\right|_{\delta_{\mu}}\left(z_{\mu}(v)\right)-J_{N}\left(\left.u\right|_{\delta_{\mu}}\left(z_{\mu}(v)\right)\right)\right)^{2} d v \\
& \quad \leqslant C N^{-2 m} \frac{h_{\mu}}{2}\left\|\partial_{v}^{m}\left(\left.u\right|_{\delta_{\mu}}\left(z_{\mu}(\cdot)\right)\right)\right\|_{L^{2}(-1,1)}^{2} \\
& \quad \leqslant C N^{-2 m}\left(\frac{h_{\mu}}{2}\right)^{2 m}\left\|\partial_{z}^{m}\left(\left.u\right|_{\delta_{\mu}}(\cdot)\right)\right\|_{L^{2}\left(\delta_{\mu}\right)}^{2} .
\end{aligned}
$$

This helps us to deduce that

$$
\begin{aligned}
\left\|u-I_{N} u\right\|_{L^{2}(-1,1)}^{2} & =\sum_{\mu=0}^{M} \int_{\eta_{\mu}}^{\eta_{\mu+1}}\left(\left.u\right|_{\delta_{\mu}}(z)-I_{N}^{\mu}\left(\left.u\right|_{\delta_{\mu}}\right)(z)\right)^{2} d z \\
& \leqslant C h^{2 m} N^{-2 m} \sum_{\mu=0}^{M}\left\|\partial_{z}^{m}\left(\left.u\right|_{\delta_{\mu}}(\cdot)\right)\right\|_{L^{2}\left(\delta_{\mu}\right)}^{2} \\
& =C h^{2 m} N^{-2 m}\left\|\partial_{z}^{m} u\right\|_{L^{2}(-1,1)}^{2},
\end{aligned}
$$

which leads to (4.6). 
Using (4.3), we have

$$
\begin{aligned}
\left\|u-I_{N} u\right\|_{L^{\infty}(-1,1)} & =\max _{0 \leqslant \mu \leqslant M}\left\{\left\|\left.u\right|_{\delta_{\mu}}\left(z_{\mu}(\cdot)\right)-J_{N}\left(\left.u\right|_{\delta_{\mu}}\left(z_{\mu}(\cdot)\right)\right)\right\|_{L^{\infty}(-1,1)}\right\} \\
& \leqslant C N^{1 / 2-m} \max _{0 \leqslant \mu \leqslant M}\left\{\left\|\partial_{v}^{m}\left(\left.u\right|_{\delta_{\mu}}\left(z_{\mu}(\cdot)\right)\right)\right\|_{L^{2}(-1,1)}\right\} \\
& =C N^{1 / 2-m} \max _{0 \leqslant \mu \leqslant M}\left\{\left(\frac{h_{\mu}}{2}\right)^{m}\left\|\partial_{v}^{m}\left(\left.u\right|_{\delta_{\mu}}\left(z_{\mu}(\cdot)\right)\right)\right\|_{L^{2}(-1,1)}\right\} \\
& =C N^{1 / 2-m} \max _{0 \leqslant \mu \leqslant M}\left\{\left(\frac{h_{\mu}}{2}\right)^{m-1 / 2}\left\|\partial_{v}^{m}\left(\left.u\right|_{\delta_{\mu}}(\cdot)\right)\right\|_{L^{2}\left(\delta_{\mu}\right)}\right\} \\
& \leqslant C h^{m-1 / 2} N^{1 / 2-m}\left\|\partial_{z}^{m} u\right\|_{L^{2}(-1,1)} .
\end{aligned}
$$

This is (4.7).

Now we begin to prove (4.8). The following derivation is clear,

$$
\left\|I_{N} u\right\|_{L^{\infty}(-1,1)}=\max _{0 \leqslant \mu \leqslant M}\left\|I_{N}^{\mu}\left(\left.u\right|_{\delta_{\mu}}\right)\right\|_{L^{\infty}\left(\sigma_{\mu}\right)} .
$$

We use (4.5) to estimate $\left\|I_{N}^{\mu}\left(\left.u\right|_{\delta_{\mu}}\right)\right\|_{L^{\infty}\left(\delta_{\mu}\right)}$ as follows,

$$
\begin{aligned}
\left\|I_{N}^{\mu}\left(\left.u\right|_{\delta_{\mu}}\right)\right\|_{L^{\infty}\left(\delta_{\mu}\right)} & =\left\|\left(I_{N}^{\mu}\left(\left.u\right|_{\delta_{\mu}}\right)\right)\left(z_{\mu}(\cdot)\right)\right\|_{L^{\infty}(-1,1)}=\left\|J_{N}\left(\left.u\right|_{\delta_{\mu}}\left(z_{\mu}(\cdot)\right)\right)\right\|_{L^{\infty}(-1,1)} \\
& \leqslant C \log (N+1)\left\|\left.u\right|_{\delta_{\mu}}\left(z_{\mu}(\cdot)\right)\right\|_{L^{\infty}(-1,1)}=C \log (N+1)\left\|\left.u\right|_{\delta_{\mu}}\right\|_{L^{\infty}\left(\delta_{\mu}\right)} \\
& \leqslant C \log (N+1)\|u\|_{L^{\infty}(-1,1)}
\end{aligned}
$$

which together with (4.17) gives that

$$
\left\|I_{N} u\right\|_{L^{\infty}(-1,1)} \leqslant C \log (N+1)\|u\|_{L^{\infty}(-1,1)} .
$$

This leads to the desired result (4.8).

Now we begin to prove (4.9). The result (4.4) is useful in the following derivation,

$$
\begin{aligned}
\left\|I_{N} u\right\|_{L^{2}(-1,1)}^{2} & =\sum_{\mu=0}^{M}\left\|I_{N}^{\mu}\left(\left.u\right|_{\delta_{\mu}}\right)\right\|_{L^{2}\left(\delta_{\mu}\right)}^{2}=\sum_{\mu=0}^{M} \frac{h_{\mu}}{2}\left\|\left(I_{N}^{\mu}\left(\left.u\right|_{\delta_{\mu}}\right)\right)\left(z_{\mu}(\cdot)\right)\right\|_{L^{2}(-1,1)}^{2} \\
& =\sum_{\mu=0}^{M} \frac{h_{\mu}}{2}\left\|J_{N}\left(\left.u\right|_{\delta_{\mu}}\left(z_{\mu}(\cdot)\right)\right)\right\|_{L^{2}(-1,1)}^{2} \\
& \leqslant C \sum_{\mu=0}^{M} \frac{h_{\mu}}{2}\left\|\left.u\right|_{\delta_{\mu}}\left(z_{\mu}(\cdot)\right)\right\|_{L^{\infty}(-1,1)}^{2} \leqslant C \sum_{\mu=0}^{M} \frac{h_{\mu}}{2}\|u\|_{L^{\infty}(-1,1)}^{2} \\
& \leqslant C\|u\|_{L^{\infty}(-1,1)}^{2}
\end{aligned}
$$

which leads to the desired result (4.9). Now we have completed the whole proof for this lemma.

Lemma $4.3[\mathbf{5}, \mathbf{2 1}]$. Assume that $u \in H^{m}(-1,1)$ for some $m \geqslant 1$ and $\varphi \in \mathcal{P}_{N}$, which denotes the space of all polynomials of degree not exceeding $N$. Then there exists a constant $C$ independent of $N \geqslant m-1$ such that

$$
\left|\int_{-1}^{1} u(x) \varphi(x) d x-\sum_{j=0}^{N} u\left(x_{j}\right) \varphi\left(x_{j}\right) \omega_{j}\right| \leqslant C N^{-m}\left\|\partial_{x}^{m} u\right\|_{L^{2}(-1,1)}\|\varphi\|_{L^{2}(-1,1)}
$$

where the $x_{j}$ are the $(N+1)$-point Legendre Gauss-Lobatto points, corresponding weight $\omega_{j}$, $j=0,1, \ldots, N$. 
Lemma 4.4 [23]. Suppose $0 \leqslant M<+\infty$. If a nonnegative integrable function $e(x)$ satisfies

$$
e(x) \leqslant v(x)+M \int_{-1}^{x} e(z) d z
$$

where $v(x)$ is also a nonnegative integrable function, then

$$
\|e(x)\|_{L^{p}(-1,1)} \leqslant C\|v(x)\|_{L^{p}(-1,1)}, \quad p=2,+\infty .
$$

\section{Convergence analysis}

This section is devoted to providing a convergence analysis for the numerical scheme. The goal is to show that the rate of convergence is exponential, that is the spectral accuracy can be obtained for the proposed approximations. Firstly, we will carry out convergence analysis in $L^{\infty}(-1,1)$ space.

TheOREM 5.1. Let $u(x)$ be the exact solution to $(2.2), u^{N}(x)$ be the approximate solution, and $\rho^{N}(x)$ be the approximate derivative obtained by using the spectral-collocation schemes (2.12) and (2.14). Then for $N \geqslant m-1$ sufficiently large,

$$
\left\|e_{i}(x)\right\|_{L^{\infty}(-1,1)} \leqslant C h^{m-1 / 2} N^{1 / 2-m}\left(\widetilde{R}\|u\|_{L^{\infty}(-1,1)}+(1+\widetilde{R})\left\|\partial_{x}^{m+1} u\right\|_{L^{2}(-1,1)}\right), \quad i=0,1,
$$

where

$$
\begin{gathered}
e_{0}(x):=u(x)-u^{N}(x), \quad x \in[-1,1], \\
e_{1}(x):=u^{\prime}(x)-\rho^{N}(x), \quad x \in[-1,1], \\
\widetilde{R}:=\max _{x \in[-1,1]}\left\|\partial_{z}^{m}(R(x, \cdot))\right\|_{L^{2}(-1, x)} .
\end{gathered}
$$

From (5.1) we can see that the convergence rate of the numerical errors decays at the rate $h^{m-1 / 2} N^{1 / 2-m}$ which depends not only on $N$ but also on $m$. This implies that if the data functions have better regularity, that is $m$ is larger, the errors decay faster. If we employ more collocation points, that is $N$ is larger, we can obtain higher accuracy. If we divide the interval $[-1,1]$ into more small subintervals, that is $h$ is smaller, the errors decay faster. It is worth mentioning that $N, h$ and $m$ are independent of each other. In theory, (5.1) shows that errors decay to zero for sufficiently large $N$ or $1 / h$, while in fact, the numerical errors will stabilize at some level near the machine precision. This result is confirmed by Figure 1 of Example 1.

Proof. Note that in each subinterval $\left(\eta_{\mu}, \eta_{\mu+1}\right], \mu=0,1, \ldots, M, \rho^{N}(s)$ is polynomial of degree not exceeding $N$ in each subinterval $\left[\eta_{\mu}, \eta_{\mu+1}\right], 0,1, \ldots, M$. Then

$$
\alpha\left(x_{i}^{\mu}\right)=\int_{-1}^{x_{i}^{\mu}} \rho^{N}(z) d z,
$$

which allows us to deduce that

$$
u\left(x_{i}^{\mu}\right)-u_{i}^{\mu}=\int_{-1}^{x_{i}^{\mu}} e_{1}(z) d z .
$$

Subtracting (2.12) from (2.5) yields

$$
u^{\prime}\left(x_{i}^{\mu}\right)-\rho_{i}^{\mu}=A\left(x_{i}^{\mu}\right) \int_{-1}^{x_{i}^{\mu}} e_{1}(z) d z+\int_{-1}^{x_{i}^{\mu}} R\left(x_{i}^{\mu}, z\right) e_{0}(z) d z+E_{1}\left(x_{i}^{\mu}\right),
$$


where

$$
E_{1}(x):=\int_{-1}^{x} R(x, z) u^{N}(z) d z-\beta(x), \quad x \in[-1,1] .
$$

Multiplying both sides of (5.3) by $F_{i}^{\mu}(x)$ and summing from $i=0$ to $N$ we obtain that

$$
\begin{aligned}
& \sum_{i=0}^{N} u^{\prime}\left(x_{i}^{\mu}\right) F_{i}^{\mu}(x)-\sum_{i=0}^{N} \rho_{i}^{\mu} F_{i}^{\mu}(x) \\
& =\sum_{i=0}^{N}\left(A\left(x_{i}^{\mu}\right) \int_{-1}^{x_{i}^{\mu}} e_{1}(z) d z\right) F_{i}^{\mu}(x)+\sum_{i=0}^{N}\left(\int_{-1}^{x_{i}^{\mu}} R\left(x_{i}^{\mu}, z\right) e_{0}(z) d z\right) F_{i}^{\mu}(x) \\
& \quad+\sum_{i=0}^{N} E_{1}\left(x_{i}^{\mu}\right) F_{i}^{\mu}(x), \quad x \in\left[\eta_{\mu}, \eta_{\mu+1}\right]
\end{aligned}
$$

By the definition of $I_{N}$ and $\rho^{N}(x)$, we have for $x \in[-1,1]$

$$
I_{N} u^{\prime}(x)-\rho^{N}(x)=I_{N}\left(A(x) \int_{-1}^{x} e_{1}(z) d z\right)+I_{N}\left(\int_{-1}^{x} R(x, z) e_{0}(z) d z\right)+I_{N} E_{1}(x)
$$

which leads to

$$
e_{1}(x)=I_{N} E_{1}(x)+\sum_{j=2}^{4} E_{j}(x)+A(x) \int_{-1}^{x} e_{1}(z) d z+\int_{-1}^{x} R(x, z) e_{0}(z) d z,
$$

where

$$
\begin{gathered}
E_{2}(x):=\left(I-I_{N}\right) u^{\prime}(x), \\
E_{3}(x):=\left(I_{N}-I\right) \int_{-1}^{x} R(x, z) e_{0}(z) d z, \\
E_{4}(x):=\left(I_{N}-I\right)\left(A(x) \int_{-1}^{x} e_{1}(z) d z\right) .
\end{gathered}
$$

Applying the Dirichlet formula to the last term in the right-hand side of (5.7) yields

$$
\int_{-1}^{x} R(x, z) e_{0}(z) d z=\int_{-1}^{x}\left[\int_{s}^{x} R(x, z) d z\right] e_{1}(s) d s
$$

which helps to deduce that there exist constants $C>0$ such that

$$
\left|A(x) \int_{-1}^{x} e_{1}(z) d z+\int_{-1}^{x} R(x, z) e_{0}(z) d z\right| \leqslant C \int_{-1}^{x} e_{1}(z) d z
$$

Then by Lemma $4.4, e_{1}(x)$ in $(5.7)$ can be estimated as

$$
\left\|e_{1}(x)\right\|_{L^{\infty}(-1,1)} \leqslant C\left(\left\|I_{N} E_{1}(x)\right\|_{L^{\infty}(-1,1)}+\sum_{j=2}^{4}\left\|E_{j}(x)\right\|_{L^{\infty}(-1,1)}\right) .
$$

We estimate each term of the right-hand side of the above inequality one by one.

First we estimate $\left\|I_{N} E_{1}(x)\right\|_{L^{\infty}(-1,1)}$. Inequality (4.8) gives that

$$
\left\|I_{N} E_{1}(x)\right\|_{L^{\infty}(-1,1)} \leqslant C \log (N+1)\left\|E_{1}(x)\right\|_{L^{\infty}(-1,1)} .
$$


We estimate $\left\|E_{1}(x)\right\|_{L^{\infty}(-1,1)}$. Note that $E_{1}(x)$ can be written as

$$
\begin{aligned}
E_{1}(x)= & \sum_{r=0}^{\mu-1}\left(\int_{\eta_{r}}^{\eta_{r+1}} R(x, z) u_{r}(z) d z-\frac{h_{r}}{2} \beta_{1}^{r}(x)\right) \\
& +\int_{\eta_{\mu}}^{x} R(x, z) u_{\mu}(z) d z-\frac{h_{\mu}}{2} \frac{x+1}{2} \beta_{2}(x), \quad x \in \delta_{\mu}, \mu \geqslant 0 .
\end{aligned}
$$

For $x \in \delta_{\mu}$,

$$
\begin{aligned}
& \left|\int_{\eta_{r}}^{\eta_{r+1}} R(x, z) u_{r}(z) d z-\frac{h_{r}}{2} \beta_{1}^{r}(x)\right| \\
& \quad \leqslant C N^{-m} \frac{h_{r}}{2}\left\|\partial_{v}^{m}\left(R\left(x, z_{r}(x, \cdot)\right)\right)\right\|_{L^{2}(-1,1)}\left\|u_{r}\left(z_{r}(\cdot)\right)\right\|_{L^{2}(-1,1)} \\
& \quad \leqslant C N^{-m}\left(\frac{h_{r}}{2}\right)^{m+1 / 2}\left\|\left.\partial_{z}^{m}(R(x, z))\right|_{z=z_{r}(\cdot)}\right\|_{L^{2}(-1,1)}\left\|u_{r}\right\|_{L^{2}\left(\delta_{r}\right)} \\
& \quad \leqslant C N^{-m}\left(\frac{h_{r}}{2}\right)^{m}\left\|\partial_{z}^{m}(R(x, \cdot))\right\|_{L^{2}\left(\delta_{r}\right)}\left\|u_{r}\right\|_{L^{2}\left(\delta_{r}\right)} \\
& \quad \leqslant C N^{-m} h^{m}\left\|\partial_{z}^{m}(R(x, \cdot))\right\|_{L^{2}\left(\delta_{r}\right)}\left\|u_{r}\right\|_{L^{2}\left(\delta_{r}\right)}
\end{aligned}
$$

Similarly,

$\left|\int_{\eta_{\mu}}^{x} R(x, z) u_{\mu}(z) d z-\frac{h_{\mu}}{2} \frac{\vartheta_{\mu}(x)+1}{2} \beta_{2}(x)\right| \leqslant C N^{-m} h^{m}\left\|\partial_{z}^{m}(R(x, \cdot))\right\|_{L^{2}\left(\eta_{\mu}, x\right)}\left\|u_{\mu}\right\|_{L^{2}\left(\delta_{\mu}\right)}$,

where

$$
\vartheta_{\mu}(x):=\frac{2}{h_{\mu}} x-\frac{\eta_{\mu+1}+\eta_{\mu}}{h_{\mu}}, \quad x \in\left[\eta_{\mu}, \eta_{\mu+1}\right] .
$$

By the Cauchy inequality, which states that

$$
\sum_{r=0}^{\mu} a_{r} b_{r} \leqslant\left(\sum_{r=0}^{\mu} a_{r}^{2}\right)^{1 / 2}\left(\sum_{r=0}^{\mu} b_{r}^{2}\right)^{1 / 2}
$$

in which we let

$$
\begin{gathered}
a_{r}=\left\|\partial_{z}^{m}(R(x, \cdot))\right\|_{L^{2}\left(\delta_{r}\right)}, \quad b_{r}=\left\|u_{r}\right\|_{L^{2}\left(\delta_{r}\right)}, \quad r=0,1, \ldots, \mu-1, \\
a_{\mu}=\left\|\partial_{z}^{m}(R(x, \cdot))\right\|_{L^{2}\left(\eta_{\mu}, x\right)}, \quad b_{\mu}=\left\|u_{\mu}\right\|_{L^{2}\left(\delta_{\mu}\right)},
\end{gathered}
$$

we have for $x \in \delta_{\mu}$

$$
\begin{aligned}
\left|E_{1}(x)\right| & \leqslant C N^{-m} h^{m}\left\|\partial_{z}^{m}(R(x, \cdot))\right\|_{L^{2}(-1, x)}\left\|u^{N}\right\|_{L^{2}(-1,1)} \\
& \leqslant C N^{-m} h^{m} \widetilde{R}\left(\left\|e_{0}\right\|_{L^{\infty}(-1,1)}+\|u\|_{L^{\infty}(-1,1)}\right) .
\end{aligned}
$$

Therefore, combining (5.16) with (5.12) gives

$$
\left\|I_{N} E_{1}(x)\right\|_{L^{\infty}(-1,1)} \leqslant C N^{-m} h^{m} \log (N+1) \widetilde{R}\left(\left\|e_{0}\right\|_{L^{\infty}(-1,1)}+\|u\|_{L^{\infty}(-1,1)}\right) .
$$


Now we begin to estimate $\left\|E_{j}(x)\right\|_{L^{\infty}(-1,1)}, j=2,3,4$. Applying (4.7) to $u^{\prime}(x)$, we have

$$
\left\|E_{2}(x)\right\|_{L^{\infty}(-1,1)} \leqslant C h^{m-1 / 2} N^{(1 / 2)-m}\left\|\partial_{x}^{m+1} u\right\|_{L^{2}(-1,1)} .
$$

Now we begin to estimate $\left\|E_{3}(x)\right\|_{L^{\infty}(-1,1)}$. For simplicity of notation, we set

$$
b(x):=\int_{-1}^{x} R(x, z) e_{0}(z) d z .
$$

Applying (4.7) with $m=1$ to $b(x)$ yields

$$
\left\|\left(I_{N}-I\right) b(x)\right\|_{L^{\infty}(-1,1)} \leqslant C h^{1 / 2} N^{-1 / 2}\left\|\partial_{x}^{1} b\right\|_{L^{2}(-1,1)} .
$$

Note that

$$
\begin{aligned}
\left|\partial_{x}^{1} b(x)\right| & =\left|R(x, x) e_{0}(x)+\int_{-1}^{x} \frac{\partial R}{\partial x}(x, z) e_{0}(z) d z\right| \\
& \leqslant\left\|e_{0}\right\|_{L^{\infty}(-1,1)}\left|\left(R(x, x)+\int_{-1}^{x} \frac{\partial R}{\partial x}(x, z) d z\right)\right| \\
& \leqslant C\left\|e_{0}\right\|_{L^{\infty}(-1,1)},
\end{aligned}
$$

which together with (5.19) yields

$$
\left\|E_{3}(x)\right\|_{L^{\infty}(-1,1)}=\left\|\left(I_{N}-I\right) b(x)\right\|_{L^{\infty}(-1,1)} \leqslant C h^{1 / 2} N^{-1 / 2}\left\|e_{0}\right\|_{L^{\infty}(-1,1)} .
$$

Similarly

$$
\left\|E_{4}(x)\right\|_{L^{\infty}(-1,1)} \leqslant C h^{1 / 2} N^{-1 / 2}\left\|e_{1}\right\|_{L^{\infty}(-1,1)} .
$$

Combining (5.11) with (5.17), (5.18), (5.21) and (5.22) yields that

$$
\begin{aligned}
\left\|e_{1}(x)\right\|_{L^{\infty}(-1,1)} \leqslant & C N^{1 / 2-m} h^{m-1 / 2}\left(\widetilde{R}\|u\|_{L^{\infty}(-1,1)}+\left\|\partial_{x}^{m+1} u\right\|_{L^{2}(-1,1)}\right) \\
& +C h^{1 / 2} N^{-1 / 2}(1+\widetilde{R})\left\|e_{0}\right\|_{L^{\infty}(-1,1)} .
\end{aligned}
$$

Now we need another relation between $\left\|e_{1}(x)\right\|_{L^{\infty}(-1,1)}$ and $\left\|e_{0}\right\|_{L^{\infty}(-1,1)}$. Multiplying both sides of (5.3) by $F_{i}^{\mu}(x)$ and summing from $i=0$ to $N$ for $\mu=0,1, \ldots, M$, we obtain that

$$
e_{0}(x)=E_{0}(x)+\left(I_{N}-I\right)\left(\int_{-1}^{x} e_{1}(s) d s\right)+\int_{-1}^{x} e_{1}(s) d s
$$

where

$$
E_{0}(x):=\left(I-I_{N}\right) u(x)
$$

Then by Lemma 4.4

$$
\left\|e_{0}\right\|_{L^{\infty}(-1,1)} \leqslant C\left(\left\|E_{0}(x)\right\|_{L^{\infty}(-1,1)}+\left\|\left(I_{N}-I\right)\left(\int_{-1}^{x} e_{1}(s) d s\right)\right\|_{L^{\infty}(-1,1)}+\left\|e_{1}\right\|_{L^{\infty}(-1,1)}\right) .
$$

Using (4.7) with $E_{0}(x)$, and applying (4.7) with $m=1$ to the middle term of the right-hand side of above inequality, we have

$$
\left\|e_{0}\right\|_{L^{\infty}(-1,1)} \leqslant C h^{m+1 / 2} N^{-m-1 / 2}\left\|\partial_{x}^{m+1} u\right\|_{L^{2}(-1,1)}+C\left\|e_{1}\right\|_{L^{\infty}(-1,1)} .
$$

Plugging the above result into the last term of (5.23) yields the desired estimate (5.1) for $e_{1}$, which in turn substituted into the last term of (5.25) yields the estimate (5.1) for $e_{0}$. 
Next, we will give the error estimate in $L^{2}(-1,1)$ space.

Theorem 5.2. Let $u(x)$ be the exact solution to $(2.2)$. Let $u^{N}(x)$ be the approximate solution, and $\rho^{N}(x)$ be the approximate derivative obtained by using the spectral-collocation schemes (2.12) and (2.14). Then, for $N \geqslant m-1$ sufficiently large,

$$
\left\|e_{i}\right\|_{L^{2}(-1,1)} \leqslant C h^{m} N^{-m} \widetilde{R}(\widetilde{R}+1)\left(\|u\|_{L^{\infty}(-1,1)}+\left\|\partial_{x}^{m+1} u\right\|_{L^{2}(-1,1)}\right), \quad i=0,1 .
$$

Proof. By Lemma 4.4, it follows from (5.7) and (5.10) that

$$
\left\|e_{1}(x)\right\|_{L^{2}(-1,1)} \leqslant C\left(\left\|I_{N} E_{1}(x)\right\|_{L^{2}(-1,1)}+\sum_{j=2}^{4}\left\|E_{j}(x)\right\|_{L^{2}(-1,1)}\right) .
$$

We estimate each term of the right of the above inequality one by one. Applying (4.9) to $E_{1}(x)$ yields

$$
\left\|I_{N} E_{1}(x)\right\|_{L^{2}(-1,1)} \leqslant C\left\|E_{1}(x)\right\|_{L^{\infty}(-1,1)} .
$$

Recalling the result (5.16) and using the result of Theorem 5.1, we obtain that

$$
\begin{aligned}
\left\|I_{N} E_{1}(x)\right\|_{L^{2}(-1,1)} & \leqslant C h^{m} N^{-m} \widetilde{R}\left(\left\|e_{0}\right\|_{L^{\infty}(-1,1)}+\|u\|_{L^{\infty}(-1,1)}\right) \\
& \leqslant C h^{m} N^{-m} \widetilde{R}(\widetilde{R}+1)\left(\|u\|_{L^{\infty}(-1,1)}+\left\|\partial_{x}^{m+1} u\right\|_{L^{2}(-1,1)}\right) .
\end{aligned}
$$

Applying (4.6) to $u^{\prime}(x)$, we have

$$
\left\|E_{2}(x)\right\|_{L^{2}(-1,1)} \leqslant C h^{m} N^{-m}\left\|\partial_{x}^{m+1} u\right\|_{L^{2}(-1,1)} .
$$

The same analysis from (5.19)-(5.21), using (4.6) in Lemma 4.2 with $m=1$ for $b(x)$, yields

$$
\left\|E_{3}\right\|_{L^{2}(-1,1)}=\left\|\left(I-I_{N}\right) b(x)\right\|_{L^{2}(-1,1)} \leqslant C h N^{-1}\left\|e_{0}\right\|_{L^{\infty}(-1,1)} .
$$

Using the estimate for $e_{0}$ in Theorem 5.1 makes the above inequality become

$$
\left\|E_{3}\right\|_{L^{2}(-1,1)} \leqslant C h^{m+1 / 2} N^{-m-1 / 2}\left(\widetilde{R}\|u\|_{\infty}+\left\|\partial_{x}^{m+1} u\right\|_{L^{2}(-1,1)}\right) .
$$

Similarly

$$
\left\|E_{4}\right\|_{L^{2}(-1,1)} \leqslant C h^{m+1 / 2} N^{-m-1 / 2}\left(\widetilde{R}\|u\|_{\infty}+\left\|\partial_{x}^{m+1} u\right\|_{L^{2}(-1,1)}\right) .
$$

Combining (5.27) with (5.29), (5.30), (5.32) and (5.33) we obtain the estimate (5.26) for $e_{1}$.

Now we begin to estimate $\left\|e_{0}\right\|_{L^{2}(-1,1)}$. Applying Lemma 4.4 with $p=2$ to (5.24) yields

$$
\left\|e_{0}\right\|_{L^{2}(-1,1)} \leqslant C\left(\left\|E_{0}(x)\right\|_{L^{2}(-1,1)}+\left\|\left(I_{N}-I\right) \int_{-1}^{x} e_{1}(s) d s\right\|_{L^{2}(-1,1)}+\left\|e_{1}\right\|_{L^{2}(-1,1)}\right) .
$$

Applying (4.6) to $E_{0}(x)$, and applying (4.6) with $m=1$ to the middle term of the right-hand side of the above inequality, we have

$$
\left\|e_{0}\right\|_{L^{2}(-1,1)} \leqslant C h^{m+1} N^{-m-1}\left\|\partial_{x}^{m+1} u\right\|_{L^{2}(-1,1)}+C\left\|e_{1}\right\|_{L^{2}(-1,1)},
$$

which leads to the estimate (5.26) for $e_{0}$ by plugging the result (5.26) for $e_{1}$ into the last term of (5.34). 
(a)

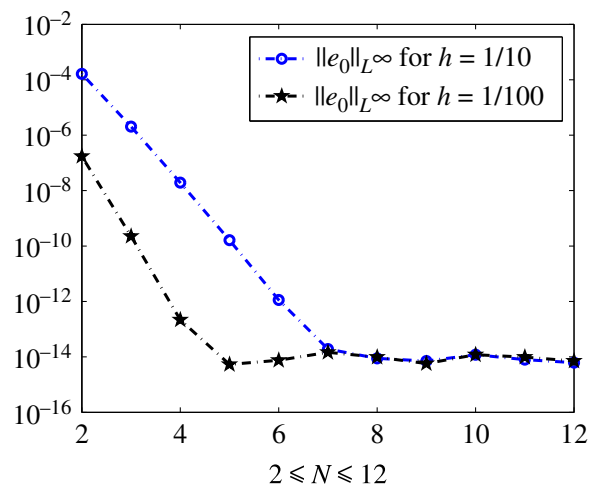

(b)

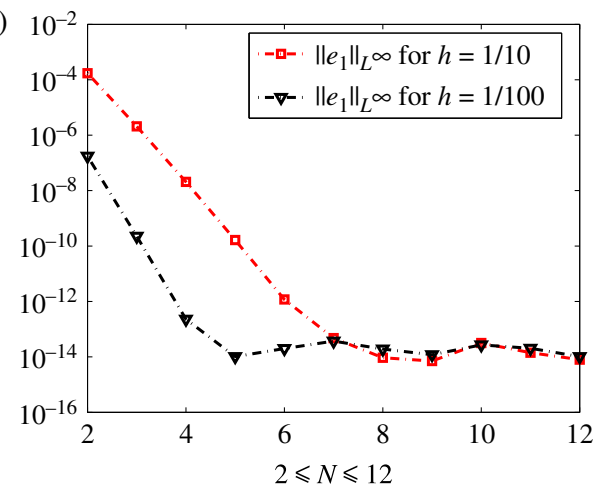

Figure 1. Example 1: (a) The errors $u-u^{N}$ versus $N$ in $L^{\infty}$ norm for $h=1 / 10$ and $h=1 / 100$. (b) The errors $u^{\prime}-\rho^{N}$ versus $N$ in $L^{\infty}$ norm for $h=1 / 10$ and $h=1 / 100$.

\section{Numerical examples}

In this section, we give three numerical examples. Example 1 is to show that the errors decay at an exponential rate and the spectral accuracy is obtained. Example 2 is to underline the role of $m$. Using the same example with $m=3$, we compare our method with the piecewise polynomial collocation method and globe Legendre spectral-collocation method. All of these examples confirm our theoretical results.

EXAmple 1. Consider (2.2) with

$$
A(x)=\sin x, \quad f(x)=e^{x}\left(1-\sin x-\left(e^{2 x}-e^{-2}\right) / 2\right), \quad R(x, z)=e^{x+z} .
$$

The corresponding exact solution is $u(x)=e^{x}, x \in[-1,1]$.

Figure 1 plots the errors for $2 \leqslant N \leqslant 12$ in $L^{\infty}$ norm with $h=1 / 10$ and $1 / 100$. The corresponding errors versus several values of $N$ are displayed in Table 1 . As expected the errors decay exponentially, which confirms our theoretical predictions. Figure 2 plots the errors with $N=5$ to underline the role of $h$. The corresponding errors versus $1 / h$ are displayed in Table 2 .

Though in theory the errors in Figure 1 should decay to zero for sufficiently large $N$, in reality they stabilize at the level $10^{-14}$ after $N>8$ because they reach the machine precision.

TABLE 1. Example 1: The errors versus $N$ in $L^{\infty}$ norm for $h=1 / 10$ and $h=1 / 100$.

\begin{tabular}{ccccccc}
\hline$N$ & 2 & 4 & 6 & 8 & 10 & 12 \\
\hline$\left\|e_{0}\right\|_{L^{\infty}}$ with $h=1 / 10$ & $1.63 \mathrm{e}-04$ & $1.93 \mathrm{e}-08$ & $1.136 \mathrm{e}-12$ & $8.88 \mathrm{e}-15$ & $1.20 \mathrm{e}-14$ & $6.22 \mathrm{e}-15$ \\
$\left\|e_{0}\right\|_{L^{\infty}}$ with $h=1 / 100$ & $1.73 \mathrm{e}-07$ & $2.17 \mathrm{e}-13$ & $7.55 \mathrm{e}-15$ & $9.77 \mathrm{e}-15$ & $1.20 \mathrm{e}-14$ & $7.11 \mathrm{e}-15$ \\
$\left\|e_{1}\right\|_{L^{\infty}}$ with $h=1 / 10$ & $1.71 \mathrm{e}-04$ & $2.07 \mathrm{e}-08$ & $1.18 \mathrm{e}-12$ & $9.33 \mathrm{e}-15$ & $3.15 \mathrm{e}-14$ & $7.99 \mathrm{e}-15$ \\
$\left\|e_{1}\right\|_{L^{\infty}}$ with $h=1 / 100$ & $1.74 \mathrm{e}-07$ & $2.28 \mathrm{e}-13$ & $2.00 \mathrm{e}-14$ & $1.91 \mathrm{e}-14$ & $2.71 \mathrm{e}-14$ & $1.02 \mathrm{e}-14$ \\
\hline
\end{tabular}

TABle 2. Example 1: The errors with $N=5$ versus $1 / h$ in $L^{\infty}$ norm.

\begin{tabular}{ccccccc}
\hline $1 / h$ & 2 & 12 & 22 & 32 & 42 & 52 \\
\hline$\left\|e_{0}\right\|_{L^{\infty}}$ & $1.69 \mathrm{e}-06$ & $5.53 \mathrm{e}-11$ & $1.51 \mathrm{e}-12$ & $1.60 \mathrm{e}-13$ & $3.02 \mathrm{e}-14$ & $8.88 \mathrm{e}-15$ \\
$\left\|e_{1}\right\|_{L^{\infty}}$ & $2.01 \mathrm{e}-06$ & $5.61 \mathrm{e}-11$ & $1.52 \mathrm{e}-12$ & $1.60 \mathrm{e}-13$ & $3.06 \mathrm{e}-14$ & $1.15 \mathrm{e}-14$ \\
\hline
\end{tabular}


(a)

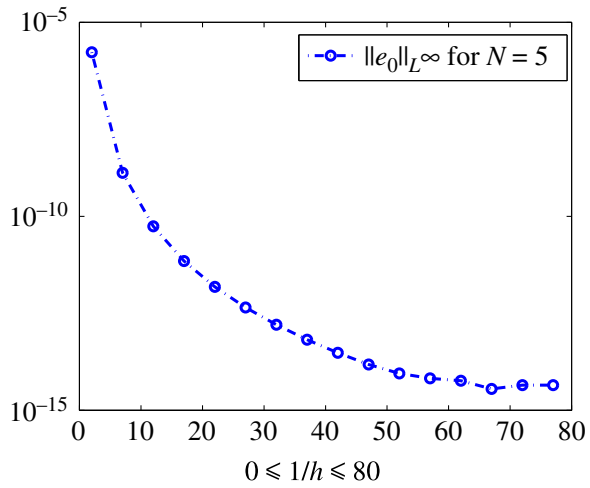

(b)

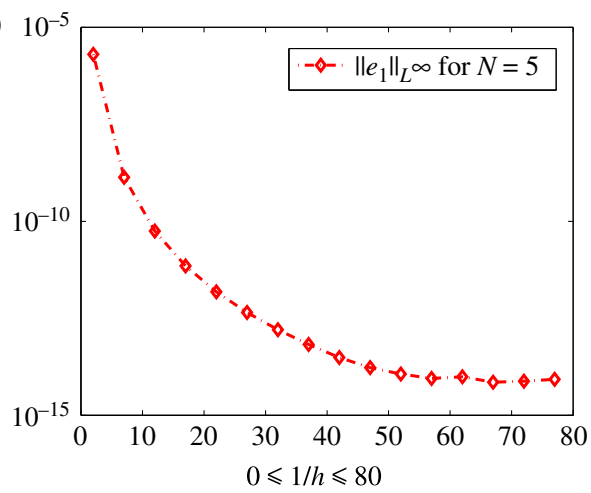

Figure 2. Example 1: (a) The errors $u-u^{N}$ with $N=5$ versus $1 / h$ in $L^{\infty}$ norm. (b) The errors $u^{\prime}-\rho^{N}$ with $N=5$ versus $1 / h$ in $L^{\infty}$ norm.

(a)

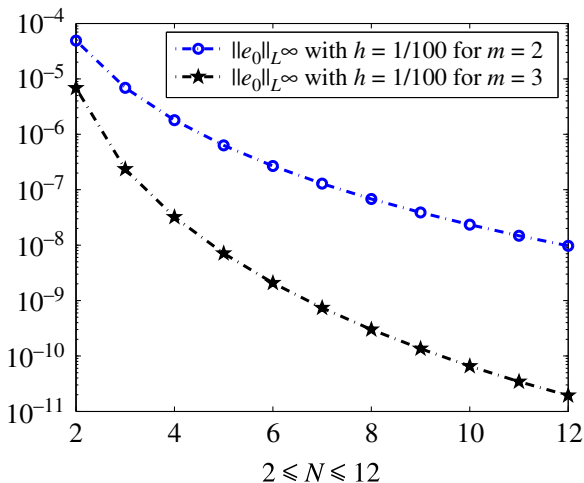

(b)

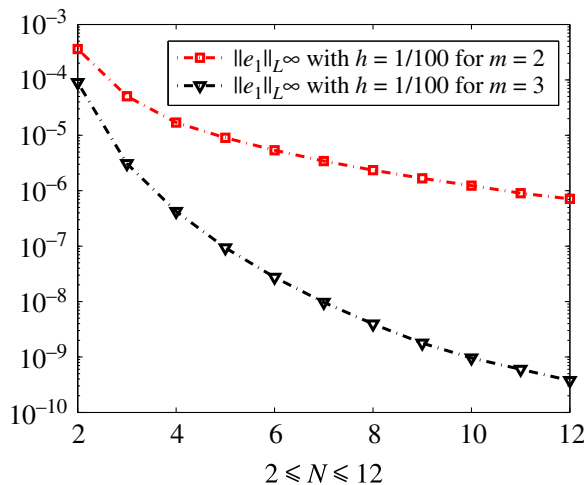

Figure 3. Example 2: (a) The errors $u-u^{N}$ with $h=1 / 100$ versus $N$ in $L^{\infty}$ norm for $m=2,3$. (b) The errors $u^{\prime}-\rho^{N}$ with $h=1 / 100$ versus $N$ in $L^{\infty}$ norm for $m=2,3$.

ExAmple 2. Consider (1.1) with

$$
\begin{gathered}
a(t)=t^{m+1 / 2}, \quad K(t, s)=t^{m+1 / 2}+s^{m+1 / 2} \\
g(t)=(m+1 / 2) t^{m-1 / 2}-t^{2 m+1}-t^{2 m+2}\left(\frac{2}{2 m+3}+\frac{1}{2 m+2}\right) .
\end{gathered}
$$

The corresponding exact solution is

$$
y(t)=t^{m+1 / 2}, \quad t \in[0,2] .
$$

It is worth noting that the solution $y(t)$ possesses the continuous $m$ th derivative while its $(m+1)$ th derivative is singular at the point $t=0$. Figure 3 plots the errors for $2 \leqslant N \leqslant 12$ in $L^{\infty}$ norm. The corresponding errors versus several values of $N$ are displayed in Table 3 .

TABLE 3. Example 2: The errors with $h=1 / 100$ versus $N$ in $L^{\infty}$ norms.

\begin{tabular}{ccccccc}
\hline$N$ & 2 & 4 & 6 & 8 & 10 & 12 \\
\hline$\left\|e_{0}\right\|_{L^{\infty}}$ for $m=2$ & $4.89 \mathrm{e}-05$ & $1.80 \mathrm{e}-06$ & $2.67 \mathrm{e}-07$ & $6.82 \mathrm{e}-08$ & $2.34 \mathrm{e}-08$ & $9.74 \mathrm{e}-09$ \\
$\left\|e_{0}\right\|_{L^{\infty}}$ for $m=3$ & $6.82 \mathrm{e}-06$ & $3.21 \mathrm{e}-08$ & $2.08 \mathrm{e}-09$ & $3.00 \mathrm{e}-10$ & $6.55 \mathrm{e}-11$ & $1.94 \mathrm{e}-11$ \\
$\left\|e_{1}\right\|_{L^{\infty}}$ for $m=2$ & $3.59 \mathrm{e}-04$ & $1.70 \mathrm{e}-05$ & $5.36 \mathrm{e}-06$ & $2.35 \mathrm{e}-06$ & $1.23 \mathrm{e}-06$ & $7.15 \mathrm{e}-07$ \\
$\left\|e_{1}\right\|_{L^{\infty}}$ for $m=3$ & $8.96 \mathrm{e}-05$ & $4.22 \mathrm{e}-07$ & $2.74 \mathrm{e}-08$ & $3.95 \mathrm{e}-09$ & $9.59 \mathrm{e}-10$ & $3.77 \mathrm{e}-10$ \\
\hline
\end{tabular}




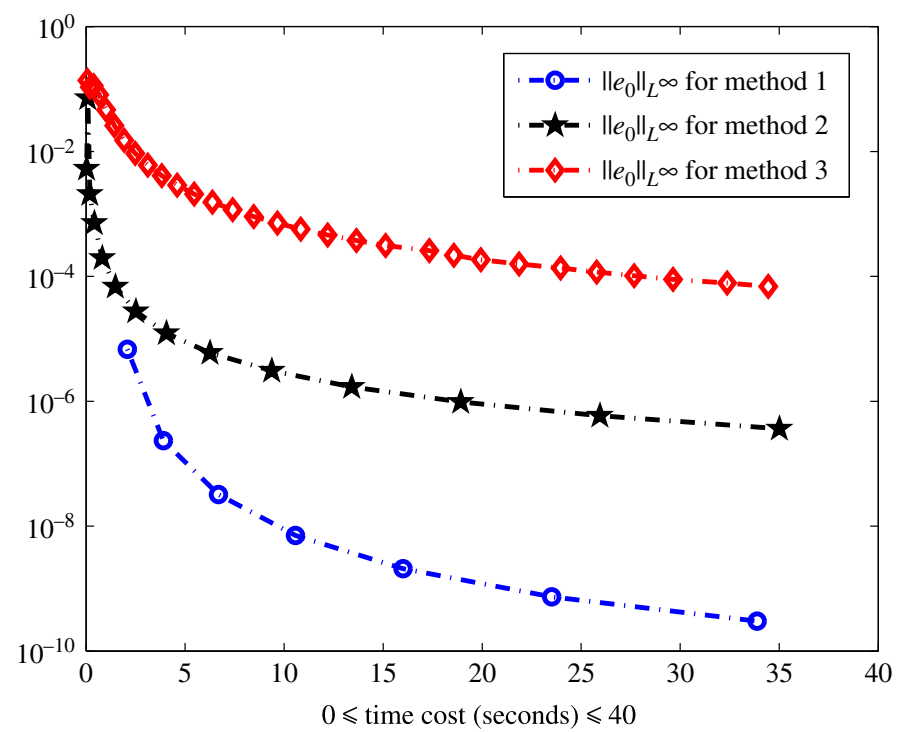

Figure 4. Example 2 with $m=3$ : The errors versus computation time cost (seconds) in $L^{\infty}$ norm for methods $1-3$.

From Figure 3 we can see that if $m$ is bigger, the errors decay faster. This coincides with our theoretical results.

In order to compare our method (method 1) with the globe Legendre spectral-collocation method (method 2) and piecewise polynomial collocation method (method 3), we fix $m=3$ in Example 2 and compute the errors by methods 1, 2 and 3 respectively. The errors versus the computation time cost for these three method are plotted in Figure 4 and displayed in Table 4. From Figure 4 we can see that using the same computation time cost our method (method 1) can obtain much higher accuracy than other two methods.

TABLE 4. Example 2 with $m=3$ : The errors $u-u^{N}$ versus computation time cost (seconds) in $L^{\infty}$ norm for methods $1-3$.

\begin{tabular}{lllllll}
\hline Time cost for method 1 & 3.90 & 6.69 & 10.57 & 16.01 & 23.51 & 33.89 \\
$L^{\infty}$-error for method 1 & $2.35 \mathrm{e}-07$ & $3.21 \mathrm{e}-08$ & $7.10 \mathrm{e}-09$ & $2.08 \mathrm{e}-09$ & $7.37 \mathrm{e}-10$ & $3.00 \mathrm{e}-10$ \\
Time cost for method 2 & 0.02 & 0.28 & 1.94 & 7.69 & 22.20 & 35.01 \\
$L^{\infty}$-error for method 2 & $5.47 \mathrm{e}-02$ & $1.68 \mathrm{e}-03$ & $4.28 \mathrm{e}-05$ & $4.26 \mathrm{e}-06$ & $7.55 \mathrm{e}-07$ & $3.65 \mathrm{e}-07$ \\
Time cost for method 3 & 0.19 & 1.67 & 5.92 & 12.99 & 23.21 & 36.20 \\
$L^{\infty}$-error for method 3 & $1.08 \mathrm{e}-01$ & $1.97 \mathrm{e}-02$ & $1.79 \mathrm{e}-03$ & $4.16 \mathrm{e}-04$ & $1.46 \mathrm{e}-04$ & $6.51 \mathrm{e}-05$ \\
\hline
\end{tabular}

\section{Conclusion and future work}

We propose the piecewise Legendre spectral-collocation method to solve the VIDEs, and provide convergence analysis for the proposed method. Numerical examples are provided to confirm the theoretical results that the numerical errors decay exponentially. We provide numerical examples to show that our method performs better than the globe Legendre spectralcollocation method and piecewise polynomial collocation method. Our method has a better convergence rate and the approximation solution can be polynomial of any high degree.

Our future work will focus on the piecewise spectral method for the Volterra functional integral and differential integral equations with delay and the system of Volterra integral equations. 


\section{References}

1. I. ALI, 'Convergence analysis of spectral methods for integro-differential equations with vanishing proportional delays', J. Comput. Math. 29 (2011) 50-61.

2. I. Ali, H. Brunner and T. TANG, 'A spectral method for pantograph-type delay differential equations and its convergence analysis', J. Comput. Math. 27 (2009) 254-265.

3. I. Ali, H. Brunner and T. TANG, 'Spectral methods for pantograph-type differential and integral equations with multiple delays', Front. Math. China 4 (2009) 49-61.

4. H. BRunner, Collocation methods for Volterra integral and related functional differential equations (Cambridge University Press, London, 2004).

5. C. Canuto, M. Y. Hussaini, A. Quarteroni and T. A. Zang, Spectral method fundamentals in single domains (Springer, 2006).

6. Y. Chen, X. Li and T. TANG, 'A note on Jacobi spectral-collocation methods for weakly singular Volterra integral equations with smooth solutions', J. Comput. Math. 31 (2013) 47-56.

7. Y. Chen and T. TANG, 'Spectral methods for weakly singular Volterra integral equations with smooth solutions', J. Comput. Appl. Math. 233 (2009) 938-950.

8. Y. Chen and T. TAng, 'Convergence analysis of the Jacobi spectral-collocation methods for Volterra integral equation with a weakly singular kernel', Math. Comput. 79 (2010) 147-167.

9. J. C. Clements and B. R. Smith, 'Parameter estimation in a reaction-diffusion model for synaptic transmission at a neuromuscular junction', Can. Appl. Math. Q. 4 (1996) 157-173.

10. A. Corduneanu and Gh. Morosanu, 'A linear integro-differential equation related to a problem from capillarity theory', Comm. Appl. Nonlinear Anal. 3 (1996) 51-60.

11. C. M. Elliot and S. MCKee, 'On the numerical solution of an integro-differential equation arising from wave-power hydraulics', BIT Numer. Math. 21 (1981) 318-325.

12. A. Goldfine, 'Taylor series methods for the solution of Volterra integral and integro-differential equations', Math. Comput. 31 (1977) 691-707.

13. Z. Gu and Y. CHEN, 'Legendre spectral-collocation method for Volterra integral equations with nonvanishing delay', Calcolo (2013) 1-24.

14. J. S. Hesthaven, 'From electrostatics to almost optimal nodal sets for polynomial interpolation in a simplex', SIAM J. Numer. Anal. 35 (1998) 655-676.

15. Y. JiAng, 'On spectral methods for Volterra-type integro-differential equations', J. Comput. Appl. Math. 230 (2009) 333-340.

16. X. Li and T. TANG, 'Convergence analysis of the Jacobi collocation methods for Abel-Volterra integral equations of the second kind', Front. Math. China 7 (2012) 69-84.

17. A. Makroglou, 'Computer treatment of the integro-differential equations of collective nonruin; the finite time case', Math. Comput. Simulation 54 (2000) 93-112.

18. P. Nevai, 'Mean convergence of Lagrange interpolation, III', Trans. Amer. Math. Soc. 282 (1984) 669-698.

19. J. M. Ortega, Numerical analysis: a second course (Academic Press, New York, 1972).

20. S. Shaw and J. R. Whiteman, 'Adaptive space-time finite element solution for Volterra equations in viscoelasticity problems', J. Comput. Appl. Math. 125 (2000) 337-345.

21. J. Shen and T. TAng, Spectral and high-order methods with applications (Science Press, Beijing, 2006).

22. T. TANG and $X$. XU, 'Accuracy enhancement using spectral postprocessing for differential equations and integral equations', Commun. Comput. Phys. 5 (2009) 779-792.

23. T. TANG, X. XU and J. Cheng, 'On Spectral methods for Volterra integral equation and the convergence analysis', J. Comput. Math. 26 (2008) 825-837.

24. A. Visintin, Differential models of hysteresis (Springer, Berlin and Heidelberg, 1994).

25. V. Volterra, 'Variazioni e fluttuazioni del numero d'indinvidui in specie animali conviventi', Memorie del R. Comitato talassografico italiano, Men. CXXXI (1927).

26. V. Volterra, Theory of functionals and of integral and integro-differential equations (Dover Publications, New York, 1959).

27. Z. WAn, Y. Chen and Y. HuAng, 'Legendre spectral Galerkin method for second-kind Volterra integral equations', Front. Math. China 4 (2009) 181-193.

28. Y. WEI and Y. CHEN, 'Convergence analysis of the Legendre spectral collocation methods for second order Volterra integro-differential equations', Numer. Math. Theory Methods Appl. 4 (2011) 419-438.

29. Y. WEI and Y. CHEN, 'Convergence analysis of the spectral methods for weakly singular Volterra integrodifferential equations with smooth solutions', Adv. Appl. Math. Mech. 4 (2012) 1-20.

30. Y. WeI and Y. CHEN, 'Legendre spectral collocation methods for pantograph Volterra delay-integrodifferential equations', J. Sci. Comput. 53 (2012) 672-688.

31. Z. XIE, X. Li and T. TANG, 'Convergence analysis of spectral Galerkin methods for Volterra type integral equations', J. Sci. Comput. 53 (2012) 414-434.

32. W. YuAn and T. TANG, 'The numerical analysis of Runge-Kutta methods for a certain nonlinear integrodifferential equation', Math. Comput. 54 (1990) 155-168.

33. J. ZhaO and R. M. Corless, 'Compact finite difference method for integro-differential equations', Appl. Math. Comput. 177 (2006) 271-288. 
Zhendong $\mathrm{Gu}$

Department of Applied Mathematics Guangdong University of Finance

Guangzhou 510521

China

guzhd@qq.com
Yanping Chen

School of Mathematics Science South China Normal University

Guangzhou 510631

China

yanpingchen@scnu.edu.cn 